УДК 539.3

DOI https://doi.org/10.26661/2413-6549-2020-2-03

\title{
НЕСТАЦІОНАРНА ЗАДАЧА ТЕПЛОПРОВІДНОСТІ ДЛЯ ШАРУВАТОЇ ПІВ БЕЗМЕЖНОЇ ПЛИТИ
}

\author{
Турчин I. М. \\ доктор фізико-математичних наук, дочент, \\ професор кафедри механіки \\ Львівський національний університет імені Івана Франка \\ вул. Університетська, 1, Львів, Україна \\ orcid.org/0000-0003-0345-1467 \\ ihorturchyn@gmail.com
}

Турчин О. Ю.

кандидат фізико-математичних наук, доиент, доцент кафедри математики і фізики

Начіональний лісотехнічний університет Украӥни

вул. Генерала Чупринки, 103, Львів, Україна

orcid.org/0000-0002-0046-3131

turchyn@nltu.edu.ua

\begin{abstract}
Ключові слова: нестаціонарна задача теплопровідності, шарувата пів безмежна плита, поліноми Лагерра, інтегральне перетворення Фур' ${ }^{\prime}$.
\end{abstract}

У багатьох задачах про поширення тепла в неоднорідних тілах слід ураховувати нестаціонарність процесу. Під час побудови точних аналітичних розв'язків просторових нестаціонарних задач теплопровідності неоднорідних тіл на дослідників чекають значні труднощі математичного характеру, пов'язані із застосуванням інтегрального перетворення Лапласа. Особливо це стосується випадків, коли одночасно 3 цим перетворенням застосовується інтегральне за просторовою змінною. У роботі до таких задач пропонується застосовувати новий метод - інтегральне перетворення Лагерра.

Розглянуто нестаціонарну задачу теплопровідності про нагрів пів безмежної плити тепловим потоком, який діє на іiі боковій поверхні. На межах поділу матеріалів плити виконуються умови ідеального теплового контакту. На нижній і верхній основах неоднорідної плити відбувається теплообмін за законом Ньютона. До рівнянь нестаціонарної теплопровідності для кожного шару, крайових умов та умов спряження застосовано спочатку інтегральне перетворення Лагерра за часовою змінною, а потім інтегральне cos-перетворення Фур'є за просторовою змінною. Як наслідок, отримано трикутні послідовності звичайних диференціальних рівнянь, у які ввійшли задані інтенсивності теплових потоків на бічній поверхні. Загальний розв’язок цих послідовностей отримано у вигляді алгебричної згортки фундаментальних розв'язків та набору сталих. Фундаментальні розв'язки трикутних послідовностей побудовано методом невизначених коефіцієнтів, а набір сталих визначено з трансформованих за Лагерром і Фур'є крайових умов та умов ідеального теплового контакту складників півсмуги у вигляді рекурентних співвідношень. Остаточний розв'язок вихідної задачі записано у вигляді ряду за поліномами Лагерра з коефіцієнтами у вигляді інтегралів Фур'є. Числовий експеримент проведено для пів безмежної плити з двостороннім покриттям і з тепловими властивостями алюмінієвого стопу та кераміки. Виявлено фізично обгрунтовані закономірності нестаціонарного поширення тепла в таких шаруватих тілах. 


\title{
UNSTEADY HEAT CONDUCTIVITY PROBLEM FOR LAYERED SEMIINFINITE PLATE
}

\author{
Turchyn I. M. \\ Doctor of Sciences in Physics and Mathematics, Associate Professor, \\ Professor at the Department of Mechanics \\ Ivan Franko National University of Lviv \\ Universytetska str., 1, Lviv, Ukraine \\ orcid.org/0000-0003-0345-1467 \\ ihorturchyn@gmail.com \\ Turchyn O. Yu. \\ Philosophy Doctor, Associate Professor, \\ Associate Professor at the Department of Mathematic and Physic \\ Ukrainian Forestry National University \\ Generala Chuprynky str., 103, Lviv, Ukraine \\ orcid.org/0000-0002-0046-3131 \\ turchyn@nltu.edu.ua
}

Key words: unsteady heat conductivity problem, moving heat load, layered semiinfinite plate, Laguerre polynomials, Fourier transform.
The study of the process of heat propagation in coated bodies is used in many engineering studies. In many cases, for more adequate modeling, the nonstationarity of the process should be taken into account. When constructing accurate analytical solutions of nonstationary problems of thermal conductivity, researchers face significant mathematical difficulties associated with the application of the Laplace integral transformation. This is especially true when the size of one of the components is much larger than the size of the others and this component is modeled by a half-boundless body, such as half-space. In this paper, we propose to apply a new method - integral Laguerre transformation. The nonstationary problem of thermal conductivity about the heating of a massive body with a covering by a heat stream, that is moving on a covering surface considers. At the boundary of the coating and the base, we have the conditions of ideal thermal contact. The basis is modeled by a half-space and a covering - a layer. The equation of nonstationary thermal conductivity and boundary conditions is applied first by the integral Laguerre transform with respect to the time variable, and then by the integral Fourier transform with respect to the spatial variable. The result is a triangular sequence of ordinary differential equations. The general solution of this sequence obtains in the form of an algebraic convolution of fundamental solutions and a set of constants. We propose to construct the fundamental solutions of triangular sequences by the method of indeterminate coefficients and to determine the set of constants from the boundary conditions transformed by Laguerre and Fourier and the conditions of ideal thermal contact of the base and coating in the form of recurrent relations. Ultimately, the final solution of the initial problem writes in the form of a series of Laguerre polynomials with coefficients in the form of Fourier integrals.

A numerical experiment for a half-space with thermal properties of aluminum alloy and a coating made of ceramics performs. Physically substantiated regularities of nonstationary heat distribution in inhomogeneous bodies under moving heat load reveals. 
Вступ. Тенденції розвитку сучасного матеріалознавства багато років сконцентровувались на отриманні гомогенних якісних сплавів й інших матеріалів, що мають певні задані властивості та експлуатаційні характеристики. Звичайні композиційні матеріали, отримані шляхом інтегрування металевої або керамічної матриці й дисперсної фази, мають синергетичні характеристики кожного 3 цих складників. Ці композити володіють рівномірно розподіленою зміцнювальною, ізолювальною або провідною фазами і їх результувальні властивості є гомогенними. Однак у багатьох сферах сучасної інженерії виникли завдання створення функціональних структур матеріалів із високим ступенем релаксації термічних напружень і великим опором окисленню, корозії й термічному удару, що вимагає отримання поєднання різних властивостей у певній анізотропії структури матеріалів [1]. Найпростішими 3 них є шаруваті структури, для яких характерна стрибкоподібна зміна фізико-механічних властивостей під час збереження неперервності розподілу фізичних полів.

Теорію і методи дослідження нестаціонарних температурних полів і фізико-механічних явищ, які їх супроводжують, викладено в багатьох роботах, огляд яких можна знайти, наприклад, у [2]. Однак слід зазначити, що використання в таких задачах класичного методу - інтегрального перетворення Лапласа - призводить до значних труднощів обчислювального характеру. Так, рівняння для визначення нулів знаменника трансформант i його коренів, які використовуються під час обернення інтегрального перетворення Лапласа, залежать від кількості шарів композиту, а в разі просторових задач - i від параметра обраного інтегрального перетворення за просторовою змінною [2]. У таких випадках спільне чисельне обернення інтегрального перетворення Лапласа й інтегрального перетворення на просторовій змінній (Фур'є, Ханкеля тощо) може не тільки істотно впливати на точність отриманих результатів, а й спотворювати якісну картину досліджуваного явища.

Метою роботи є розроблення ефективної аналітичної методики побудови розв'язку нестаціонарних задач теплопровідності для пів безмежних шаруватих тіл, яка грунтується на використанні інтегрального перетворення Лагерра $[4 ; 5 ; 6]$.

Формулювання задачі. Розглянемо пів безмежну плиту $0 \leq x<\infty, 0 \leq y \leq H$, яка складається $3 M$ шарів різної товщини $h_{i}\left(\sum_{i=1} h_{i}=H\right)$ і $з$ різними коефіцієнтами теплопровідності $\lambda_{T}^{(i)}$ і температуропровідності $a_{T}^{(i)}$. Починаючи з моменту часу $t=0$, плита нагрівається на бічній поверхні $x=0$ потоком тепла інтенсивності $q(t)$, а на граничних поверхнях $y=0, y=H$ відбувається теплообмін за законом Ньютона із зовнішнім середовищем нульової температури.
Розглянемо знерозмірені змінні й величини $\alpha=x / H, \gamma=y / H, \tau=a_{T}^{(0)} t / H^{2}, \gamma_{i}=h_{i} / H$, $\mathrm{Bi}_{i}=\kappa_{i} H^{\prime} / \lambda_{T}^{(i)}, \quad \tilde{a}_{T}^{(i)}=a_{T}^{(0)} / a_{T}^{(i)}, \quad \tilde{\lambda}_{T}^{(i)}=\lambda_{T}^{(i)} / \lambda_{T}^{(0)}$, $\tilde{q}(\tau)=q\left(H^{2} \tau / a_{T}^{(0)}\right) H / \lambda_{T}^{(0)}$, де $\kappa_{i}-$ коефіцієнт тепловіддачі з поверхні $y=0 \quad(i=1)$ і $з$ поверхні $y=H \quad(i=M)$, а $a_{T}^{(0)}$ і $\lambda_{T}^{(0)}-$ розмірні величини, які визначаються згідно завдань числового аналізу відповідно.

Уважаючи, що між складниками плити виконуються умови ідеально теплового контакту, температурне поле $T^{(i)}(\alpha, \gamma, \tau), i=1,2, \ldots, M$ в кожному шарі визначимо 3 рівнянь нестаціонарної теплопровідності

$$
\partial_{\alpha \alpha}^{2} T^{(i)}+\partial_{\gamma}^{2} T^{(i)}=\tilde{a}_{T}^{(i)} \partial_{\tau} T^{(i)}, \quad i=1,2, \ldots, M ;
$$

за нульових початкових умов

$$
T^{(i)}(\alpha, \gamma, 0)=0, \quad i=1,2, \ldots, M,
$$

за крайових умов

$$
\begin{gathered}
\tilde{\lambda}_{T}^{(i)} \partial_{\alpha} T^{(i)}=-\tilde{q}(\tau), \quad \alpha=0, i=1,2, \ldots, M ; \\
\partial_{\gamma} T^{(1)}-\mathrm{Bi}_{1} T^{(1)}=0, \quad \gamma=0 ; \\
\partial_{\gamma} T^{(M)}+\mathrm{Bi}_{M} T^{(M)}=0, \quad \gamma=1,
\end{gathered}
$$

за умов спряження шарів

$$
\begin{aligned}
& T^{(i)}=T^{(i+1)} ; \quad \tilde{\lambda}_{T}^{(i)} \partial_{\gamma} T^{(i)}=\tilde{\lambda}_{T}^{(i+1)} \partial_{\gamma} T^{(2)}, \\
& \gamma=\gamma_{i}, \quad i=1,2, \ldots, M-1
\end{aligned}
$$

Побудова розв'язку задачі. Застосуємо до рівняння (1) інтегральне перетворення Лагерра [5] та $\cos$-перетворення Фур'є [3]. Ураховуючи нульові початкові умови (2) та умови на поверхні $\alpha=0$ (3), одержимо послідовності диференційних рівнянь:

$$
\begin{aligned}
& \partial_{\alpha \alpha}^{2} \bar{T}_{n}^{(i)}-\left(\xi^{2}+\lambda \tilde{a}_{T}^{(i)}\right) \bar{T}_{n}^{(i)}=\lambda \tilde{a}_{T}^{(i)} \sum_{m=0}^{n-1} \bar{T}_{m}^{(i)}-\tilde{q}_{n}^{(i)}, \\
& n=0,1,2, \ldots, \quad i=1,2, \ldots, M
\end{aligned}
$$

де

$\bar{T}_{n}^{(i)}(\xi, \gamma)=\int_{0}^{\infty}\left[\int_{0}^{\infty} \exp (-\lambda \tau) T^{(i)}(\alpha, \gamma, \tau) L_{n}(\lambda \tau) d \tau\right] \cos (\xi \alpha) d \alpha$ - трансформанти за Лагерром і Фур'є, $\tilde{q}_{n}^{(i)}=\tilde{q}_{n} / \tilde{\lambda}_{T}^{(i)}$,

$\tilde{q}_{n}=\int_{0}^{\infty} \exp (-\lambda \tau) \tilde{q}(\tau) L_{n}(\lambda \tau) d \tau$ - трансформанта за Лагерром.

Загальний розв'язок послідовностей (6) має такий вигляд:

$$
\bar{T}_{n}^{(i)}(\xi, \gamma)=\sum_{j=0}^{n}\left[A_{n-j}^{(i)}(\xi) G_{j}^{(i)}(\xi, \gamma)+B_{n-j}^{(i)}(\xi) W_{j}^{(i)}(\xi, \gamma)\right]+Q_{n}^{(i)},
$$

де $G_{j}^{(i)}(\xi, \gamma), W_{j}^{(i)}(\xi, \gamma)$ - лінійно незалежні фундаментальні розв' язки однорідних послідовностей:

$$
\begin{aligned}
& d_{r y}^{2} G_{j}^{(i)}-\left(\xi^{2}+\lambda \tilde{a}_{i}\right) G_{j}^{(i)}=\lambda \tilde{a}_{T}^{(i)} \sum_{m=0}^{j-1} G_{m}^{(i)}, \\
& d_{r y}^{2} W_{j}^{(i)}-\left(\xi^{2}+\lambda \tilde{a}_{i}\right) W_{j}^{(i)}=\lambda \tilde{a}_{T}^{(i)} \sum_{m=0}^{j-1} W_{m}^{(i)}
\end{aligned}
$$

а $Q_{n}^{(i)}$ - частковий розв'язок неоднорідної послідовності, яка, враховуючи те, що $\tilde{q}_{n}^{(i)}$ не залежить 
від змінної $\gamma$, має вигляд рекурентного рівняння

$$
Q_{n}^{(i)}=\frac{1}{\xi^{2}+\lambda \tilde{a}_{T}^{(i)}}\left[\tilde{q}_{n}^{(i)}-\lambda \tilde{a}_{T}^{(i)} \sum_{m=0}^{n-1} Q_{m}^{(i)}\right], n=0,1,2, \ldots, i=1,2,
$$

розв'язок якого, використовуючи метод математичної індукції, можна знайти у вигляді

$$
Q_{n}^{(i)}=\frac{1}{\xi^{2}+\lambda \tilde{a}_{T}^{(i)}}\left\{\tilde{q}_{n}^{(i)}-\frac{\lambda \tilde{a}_{T}^{(i)}}{\xi^{2}+\lambda \tilde{a}_{T}^{(i)}} \sum_{k=0}^{n-1}\left(\frac{\xi^{2}}{\xi^{2}+\lambda \tilde{a}_{T}^{(i)}}\right)^{n-1-k} \tilde{q}_{k}^{(i)}\right\} .
$$

Загальні розв’язки однорідних послідовностей (8) (згідно з методом невизначених коефіцієнтів) можна подати у вигляді

$$
\begin{aligned}
& G_{j}^{(i)}(\xi, \gamma)=\exp \left(-\omega_{i} \gamma\right) \sum_{k=0}^{j} a_{j, k}^{(i)} \frac{\left(\omega_{i} \gamma\right)^{k}}{k !}, \\
& W_{j}^{(i)}(\xi, \gamma)=\exp \left(\omega_{i} \gamma\right) \sum_{k=0}^{j} a_{j, k}^{(i)} \frac{\left(-\omega_{i} \gamma\right)^{k}}{k !},
\end{aligned}
$$

де введено позначення $\omega_{i}=\sqrt{\xi^{2}+\lambda \tilde{a}_{T}^{(i)}}$.

Безпосередня підстановка розв'язків (11) у послідовності (8) приводить до рекурентних співвідношень щодо $a_{j, k}^{(i)}$ :

$$
a_{j, k+1}^{(i)}=0.5\left(a_{j, k+2}^{(i)}-\frac{\lambda \tilde{a}_{i}}{\omega_{i}^{2}} \sum_{m=k}^{j-1} a_{m, k}^{(i)}\right) .
$$

за довільних $a_{j, 0}^{(i)}$ i $a_{j, k}^{(i)} \equiv 0$ при $k>j$.

Для знаходження невідомих $A_{k}^{(i)}(\xi)$ і $B_{k}^{(i)}(\xi)$, які входять у розв'язок (7), використаємо крайові умови (4) й умови спряження (5), які після застосування до них інтегральних перетворень Лагерра і Фур'є матимуть такий вигляд:

$$
\begin{gathered}
d_{\gamma} \bar{T}_{n}^{(1)}-\mathrm{Bi}_{1} \bar{T}_{n}^{(1)}=0, \quad \gamma=0 ; \\
d_{\gamma} \bar{T}_{n}^{(M)}+\mathrm{Bi}_{M} \bar{T}_{n}^{(M)}=0, \quad \gamma=1 \\
\bar{T}_{n}^{(i)}=\bar{T}_{n}^{(i+1)} ; \quad \tilde{\lambda}_{T}^{(i)} d_{\gamma} \bar{T}_{n}^{(i)}=\tilde{\lambda}_{T}^{(i+1)} d_{\gamma} \bar{T}_{n}^{(i+1)}, \\
\gamma=\gamma_{i}, \quad i=1,2, \ldots, M-1
\end{gathered}
$$

Покладемо в рекурентних співвідношеннях (12) $a_{0,0}^{(i)}=1, a_{j, 0}^{(i)}=0, j=1,2, \ldots$, тоді

$$
\begin{aligned}
G_{0}^{(i)}(\xi, \gamma) & =\exp \left(-\omega_{i} \gamma\right), d_{\gamma} G_{0}^{(i)}(\xi, \gamma)=-\omega_{i} \exp \left(-\omega_{i} \gamma\right), \\
W_{0}^{(i)}(\xi, \gamma) & =\exp \left(\omega_{i} \gamma\right), d_{\gamma} W_{0}^{(i)}(\xi, \gamma)=\omega_{i} \exp \left(\omega_{i} \gamma\right)
\end{aligned}
$$

і розглянемо, враховуючи (7), першу умову (13):

$$
\sum_{j=0}^{n}\left[\begin{array}{l}
A_{n-j}^{(1)}(\xi)\left(d_{\gamma} G_{j}^{(1)}(\xi, 0)-\mathrm{Bi}_{1} G_{j}^{(1)}(\xi, 0)\right)+ \\
+B_{n-j}^{(1)}(\xi)\left(d_{\gamma} W_{j}^{(1)}(\xi, 0)-\mathrm{Bi}_{1} W_{j}^{(1)}(\xi, 0)\right)
\end{array}\right]+Q_{n}^{(1)}=0 \cdot
$$

Залишимо в лівій частині цих співвідношень невідомі доданки 3 індексом $n$, а решту перенесемо в праву частину. Ураховуючи подання (11) i (15), одержимо таку послідовність:

$$
\begin{gathered}
A_{n}^{(1)}(\xi)\left(-\omega_{1}-\mathrm{Bi}_{1}\right)+B_{n}^{(1)}(\xi)\left(\omega_{1}-\mathrm{Bi}_{1}\right)= \\
=-\sum_{j=1}^{n}\left[\begin{array}{c}
A_{n-j}^{(1)}(\xi)\left(d_{\gamma} G_{j}^{(1)}(\xi, 0)-\mathrm{Bi}_{1} G_{j}^{(1)}(\xi, 0)\right)+ \\
+B_{n-j}^{(1)}(\xi)\left(d_{\gamma} W_{j}^{(1)}(\xi, 0)-\mathrm{Bi}_{1} W_{j}^{(1)}(\xi, 0)\right)
\end{array}\right]-Q_{n}^{(1 .}
\end{gathered}
$$

Вчиняючи так само з умовами спряження (14) та другою умовою (13), одержимо послідовності систем рівнянь, які можна записати в матричному вигляді

$$
\begin{aligned}
& {\left[b_{k, l}\right]\left\{A_{n}^{(1)}, B_{n}^{(1)}, \ldots, A_{n}^{(l)}, B_{n}^{(l)}, \ldots, A_{n}^{(M)}, B_{n}^{(M)}\right\}^{\mathrm{T}}=} \\
& \left\{c_{k, n}\right\}^{\mathrm{T}}, \quad n=0,1,2, \ldots
\end{aligned}
$$

Матриця $\left[b_{k, l}\right]$ має блокову структуру, а їі коефіцієнти, що не дорівнюють нулю, та стовбець вільних членів мають такий вигляд:

$$
\begin{aligned}
& b_{1,1}=-\omega_{1}-\mathrm{Bi}_{1} ; b_{1,2}=\omega_{1}-\mathrm{Bi}_{1} ; b_{2 M-1,2 M}= \\
& =-\omega_{M}+\mathrm{Bi}_{2} ; b_{2 M, 2 M}=\omega_{M}+\mathrm{Bi}_{2} \\
& b_{2 i, 2 i-1}=\exp \left(-\omega_{i} \gamma_{i}\right) ; \quad b_{2 i, 2 i}=\exp \left(\omega_{i} \gamma_{i}\right) \text {; } \\
& b_{2 i, 2 i+1}=-\exp \left(-\omega_{i+1} \gamma_{i}\right) ; \quad b_{2 i, 2 i+2}=-\exp \left(\omega_{i+1} \gamma_{i}\right) \\
& b_{2 i+1,2 i-1}=-\tilde{\lambda}_{T}^{(i)} \omega_{i} \exp \left(-\omega_{i} \gamma_{i}\right) ; \quad b_{2 i+1,2 i}=\tilde{\lambda}_{T}^{(i)} \omega_{i} \exp \left(\omega_{i} \gamma_{i}\right) \text {; } \\
& b_{2 i+1,2 i+1}=\tilde{\lambda}_{T}^{(i+1)} \omega_{i+1} \exp \left(-\omega_{i+1} \gamma_{i}\right) \text {; } \\
& b_{2 i, 2 i+2}=\tilde{\lambda}_{T}^{(i+1)} \omega_{i+1} \exp \left(-\omega_{i+1} \gamma_{i}\right), i=\overline{1, M-1}, \\
& c_{1, n}=-\sum_{j=1}^{n}\left[\begin{array}{l}
A_{n-j}^{(1)}(\xi)\left(d_{\gamma} G_{j}^{(1)}(\xi, 0)-\mathrm{Bi}_{1} G_{j}^{(1)}(\xi, 0)\right)+ \\
+B_{n-j}^{(1)}(\xi)\left(d_{\gamma} W_{j}^{(1)}(\xi, 0)-\mathrm{Bi}_{1} W_{j}^{(1)}(\xi, 0)\right)
\end{array}\right]-Q_{n}^{(1)} \\
& c_{2 M, n}=-\sum_{j=1}^{n}\left[A_{n-j}^{(M)}(\xi)\left(d_{\gamma} G_{j}^{(M)}\left(\xi, \gamma_{M}\right)+\mathrm{Bi}_{M} G_{j}^{(M)}\left(\xi, \gamma_{M}\right)\right)+\right. \\
& \left.+B_{n-j}^{(M)}(\xi)\left(d_{\gamma} W_{j}^{(M)}\left(\xi, \gamma_{M}\right)+\mathrm{Bi}_{M} W_{j}^{(M)}\left(\xi, \gamma_{M}\right)\right)\right] ; \\
& c_{2 i, n}=-\sum_{j=1}^{n}\left[A_{n-j}^{(i)}(\xi) G_{j}^{(i)}\left(\xi, \gamma_{i}\right)+B_{n-j}^{(i)}(\xi) W_{j}^{(i)}\left(\xi, \gamma_{i}\right)\right]+ \\
& +\sum_{j=1}^{n}\left[A_{n-j}^{(i+1)}(\xi) G_{j}^{(i+1)}\left(\xi, \gamma_{i}\right)+B_{n-j}^{(i+1)}(\xi) W_{j}^{(i+1)}\left(\xi, \gamma_{i}\right)\right] ; \\
& c_{2 i+1, n}=-\tilde{\lambda}_{T}^{(i)} \sum_{j=1}^{n}\left[A_{n-j}^{(i)}(\xi) d_{\gamma} G_{j}^{(i)}\left(\xi, \gamma_{i}\right)+B_{n-j}^{(i)}(\xi) d_{\gamma} W_{j}^{(i)}\left(\xi, \gamma_{i}\right)\right]+ \\
& c_{2 i+1, n}=-\tilde{\lambda}_{T}^{(i)} \sum_{j=1}^{n}\left[A_{n-j}^{(i)}(\xi) d_{\gamma} G_{j}^{(i)}\left(\xi, \gamma_{i}\right)+B_{n-j}^{(i)}(\xi) d_{\gamma} W_{j}^{(i)}\left(\xi, \gamma_{i}\right)\right]+ \\
& +\tilde{\lambda}_{T}^{(i+1)} \sum_{j=1}^{n}\left[A_{n-j}^{(i+1)}(\xi) d_{\gamma} G_{j}^{(i+1)}\left(\xi, \gamma_{i}\right)+B_{n-j}^{(i+1)}(\xi) d_{\gamma} W_{j}^{(i+1)}\left(\xi, \gamma_{i}\right)\right], i=\overline{1, M-1} \\
& c_{n, 4}=-\mathrm{Bi}_{2} Q_{n}^{(i)}-\bar{q}_{n}^{(2)}(\xi)-\sum_{j=1}^{n}\left[A_{n-j}^{(2)}(\xi)\left(d_{\gamma} G_{j}^{(2)}(\xi, 1)+\mathrm{Bi}_{2} G_{j}^{(2)}(\xi, 1)\right)+\right. \\
& \left.+B_{n-j}^{(2)}(\xi)\left(d_{\gamma} W_{j}^{(2)}(\xi, 1)-\mathrm{Bi}_{2} W_{j}^{(2)}(\xi, 1)\right)\right] ;
\end{aligned}
$$

Iз використанням алгоритму Гаусса системи (16) приведено в трикутний вигляд та одержано їх рекурентний розв'язок

$$
\begin{gathered}
B_{n}^{(M)}=\frac{c_{2 M, n}^{*}}{b_{2 M, 2 M}^{*}}, A_{n}^{(M)}=\frac{1}{b_{2 M, 2 M}^{*}}\left(c_{2 M-1, n}^{*}-B_{n}^{(M)} b_{2 M-1,2 M}^{*}\right) ; \\
B_{n}^{(i)}=\frac{1}{b_{2 i, 2 i}^{*}}\left(c_{2 i, n}^{*}-B_{n}^{(i+1)} b_{2 i, 2 i+2}^{*}-A_{n}^{(i+1)} b_{2 i, 2 i+1}^{*}\right), \\
A_{n}^{(i)}=\frac{1}{b_{2 i-1,2 i-1}^{*}}\left(c_{n, 2 i-1}^{*}-B_{n}^{(i)} b_{2 i-1,2 i}^{*}\right), i=\overline{M-1,1},(18)
\end{gathered}
$$

де нові коефіцієнти пов'язані зі старими рекурентними співвідношеннями.

Остаточний розв'язок задачі (1)-(5) (згідно 3 формулою обернення інтегрального перетворення Лагерра [5] і формулою обернення cos-перетворення Фур'є [3]) має такий вигляд: 


$$
T^{(i)}(\alpha, \gamma, \tau)=\lambda \frac{2}{\pi} \sum_{n=0}^{\infty} L_{n}(\lambda \tau) \int_{0}^{\infty} \bar{T}_{n}^{(i)}(\xi, \gamma) \cos (\xi \alpha) d \xi .
$$

Числові результати. За формулою (20) проводився розрахунок температурного поля в тришаровій плиті у випадку, коли інтенсивність потоку тепла на торцевій поверхні задається виразом $\tilde{q}(\tau)=q^{*}\left(1-\exp \left(-\tau_{0} \tau\right)\right)$, де $q^{*}-$ величина, що має розмірність температури, а $\tau_{0}$ визначає час виходу теплового навантаження на стаціонарне. Тоді $\tilde{q}_{n}=\tilde{q}^{*}\left[\delta_{0 n} / \lambda-\left(\tau_{0}\right)^{n} /\left(\lambda+\tau_{0}\right)^{n+1}\right]$, де $\delta_{0 n}$ - символ Кронекера.

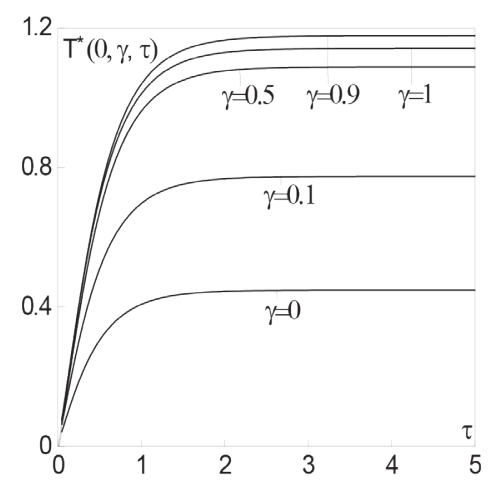

Рис. 1. Температура точок бічної поверхні плити при $\lambda_{T}^{(1)} / \lambda_{T}^{(2)}=a_{T}^{(1)} / a_{T}^{(2)}=0.5$

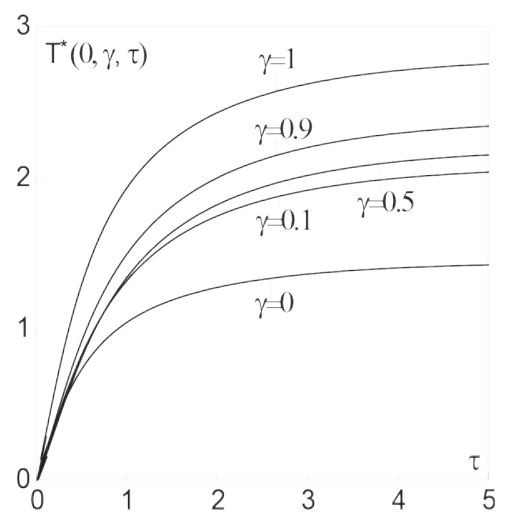

Рис. 2. Температура точок бічної поверхні плити при $\lambda_{T}^{(1)} / \lambda_{T}^{(2)}=a_{T}^{(1)} / a_{T}^{(2)}=0.1$

Теплофізичні властивості покриттів і основ, на які вони наносяться, на практиці можуть сильно відрізнятися [2]. У роботі під час чисельних розрахунків розглядалася фізично i геометрично симетрична тришарова плита: $\lambda_{T}^{(1)}=\lambda_{T}^{(3)}, a_{T}^{(1)}=a_{T}^{(3)}, h_{1}=h_{3}$, а також два варіанти співвідношення теплофізичних властивостей покриття й основи: $\lambda_{T}^{(1)} / \lambda_{T}^{(2)}=a_{T}^{(1)} / a_{T}^{(2)}=0.5$ i $\lambda_{T}^{(1)} / \lambda_{T}^{(2)}=a_{T}^{(1)} / a_{T}^{(2)}=0.1$, які дають можливість визначити характерні особливості трансформації температурного поля в градієнтних композитних плитах і вплив зміни їх градієнтних властивостей на розподіл температур.
На рисунках 1 і 2 показано результати розрахунку залежності від часової змінної $\tau$ знерозміреної температури $T^{*}(\alpha, \gamma, \tau)=T^{(i)}(\alpha, \gamma, \tau) / \tilde{q}^{*}$ точок на бічній поверхні плити при $\mathrm{Bi}_{1}=10, \mathrm{Bi}_{M}=1, \tau_{0}=4$ для першого (рис. 1) і другого (рис. 2) варіантів співвідношення теплофізичних властивостей. При цьому (за знерозмірювальні величини $\lambda_{T}^{(0)}$ i $a_{T}^{(0)}$ ) обрано відповідні величини серединного шару.

Як видно з рисунків, зниження відносних теплових властивостей покриттів призводить до зростання температури у всіх точках поверхні нагрівання і до збільшення тривалості перехідного періоду (періоду виходу на стаціонарний стан). Крім того, якщо для другого варіанта співвідношення теплофізичних властивостей температура поступово підвищується на глибині плити (від поверхні з більш інтенсивним теплообміном до поверхні з менш інтенсивним теплообміном), то для першого варіанта температура граничної поверхні $\gamma=1$ нижче за температуру поверхні поділу матеріалів покриття й основи $\gamma=0.9$.

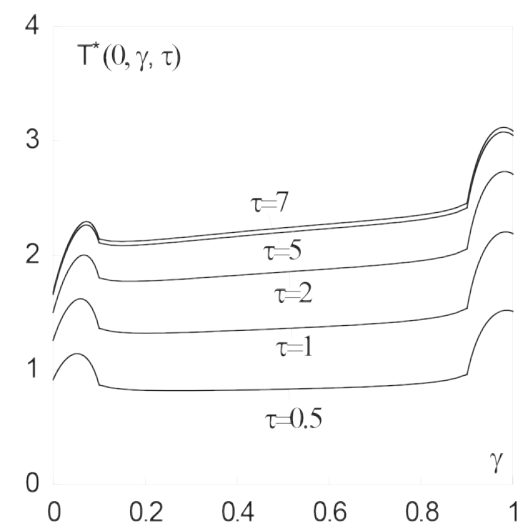

\section{Рис. 3. Температура бічної поверхні плити в різні моменти часу}

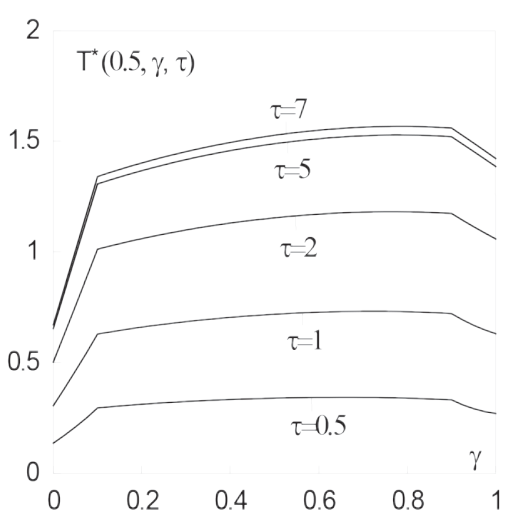

Рис. 4. Температура поверхні $\alpha=0.5$ в різні моменти часу 
На рисунках 3 і 4 показано результати розрахунку безрозмірної температури на торцевій поверхні плити і поверхні, яка перебуває від неї на відстані половини товщини плити в різні моменти безрозмірного $\tau$ часу при $\lambda_{T}^{(1)} / \lambda_{T}^{(2)}=a_{T}^{(1)} / a_{T}^{(2)}=0.1$. Як слідує 3 рисунків, процеси нагрівання поверхонь, розташованих безпосередньо під джерелами тепла і на деякій відстані від них, якісно і кількісно piзні. Безпосередня близькість джерел тепла викликає найбільш інтенсивне підвищення температури в покриттях, із віддаленням від поверхні нагрівання температура в них різко знижується, а сам процес нагріву покриттів у глибині плити здійснюється за рахунок теплопередачі від основи плити.

Насамкінець хочеться зазначити, що наведені числові результати, безумовно, не претендують на детальний аналіз температурного поля в плиті
3 покриттями і з'ясування впливу кожного геометричного і теплофізичного параметра на розподіл температури, а слугують ілюстрацією можливостей реалізації запропонованої методики побудови розв'язків двовимірних нестаціонарних задач теплопровідності пів безмежних шаруватих тіл.

Висновки. Таким чином, у роботі отримано точний аналітичний розв'язок плоскої нестаціонарної задачі теплопровідності для шаруватої напів безмежної плити, що нагрівається на бічній (торцьовій) частині поверхні змінним у часі тепловим потоком. Розв' язок отримано з використанням інтегрального перетворення Лагерра за часовою змінною та інтегрального перетворення Фур'є у вигляді ряду за поліномами Лагерра. Коефіцієнти цього ряду знаходяться із рекурентних співвідношень. Наведено результати тестового прикладу.

\section{ЛІТЕРАТУРА}

1. Tamarin Y. Protective coatings for turbine blades: monograph. USA : ASM International, 2002. 248 p.

2. Коляно Ю.М. Методы теплопроводности и термоупругости неоднородного тела : монография. Киев : Наук. думка, 1992. 280 с.

3. Sneddon I. Fourie transforms: monograph. New York : McCraw-Hill Book Company, 1951. 542 p.

4. Galazyuk V.A., Turchyn I.M. Quasistatic thermal stress state of a layer with mixed heating conditions. International Applied Mechanics. 1998. V. 34. No 9. P. 886-893.

5. Sulym H.T., Turchyn I.M. Axisymmetric quasistatic thermal stressed state in a half space with coating. Journal of Mathematical Science. 2014. V. 198, No. 1. P. 103-117.

6. Turchin I.M., Timar I., Kolodiy Yu.O. Nonstationary axisymmetric temperature field in a two-layer slab under mixed heating conditions. Journal of Engineering Physics and Thermophysics. 2015. Vol.88. Iss. 5. P. $1135-1144$.

\section{REFERENCES}

1. Tamarin Y. (2002). Protective coatings for turbine blades. ASM International, USA.

2. Kolyano, Yu. M. (1992). Metody teploprovodnosti i termouprugosti neodnorodnogo tela. [Methods of thermal conductivity and thermoelasticity of an inhomogeneous body]. Kiev: Naukova Dumka. (In Russian)

3. Sneddon, I. (1951). Fourier transforms. McCraw-Hill Book Company. New York.

4. Galazyuk, V.A. \& Turchin I.M. (1998). Quasistatic thermal stress state of a layer with mixed heating conditions. International Applied Mechanics, Volume 34, No 9, pp. 886-893.

5. Sulym H.T. \& Turchyn I.M. (2014). Axisymmetric quasistatic thermal stressed state in a half space with coating. Journal of Mathematical Science, Volume 198, No. 1. pp. 103-117.

6. Turchin, I.M., Timar, I. \& Kolodiy, Yu.O. (2015). Nonstationary axisymmetric temperature field in a two-layer slab under mixed heating conditions. Journal of Engineering Physics and Thermophysics, Volume 88, Issue 5, pp. 1135-1144. 